\title{
Phase II study of weekly vinorelbine and 24-h infusion of high-dose 5 -fluorouracil plus leucovorin as first-line treatment of advanced
} breast cancer

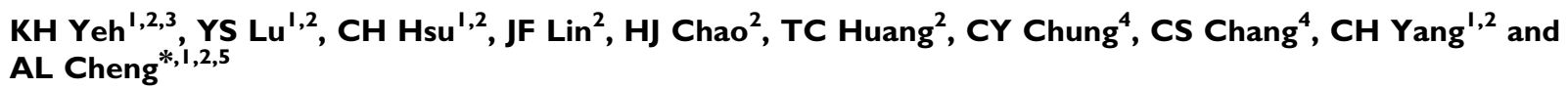

'National Taiwan University Hospital, Taiwan; ${ }^{2}$ National Taiwan University College of Medicine, Taiwan; ${ }^{3}$ Far Eastern Memorial Hospital, Taiwan;

${ }^{4}$ Changhua Christian Hospital, Taiwan; ${ }^{5}$ National Health Research Institutes, Taiwan

We prospectively investigated the efficacy and safety of combining weekly vinorelbine (VNB) with weekly 24-h infusion of high-dose 5 -fluorouracil (5-FU) and leucovorin (LV) in the treatment of patients with advanced breast cancer (ABC). Vinorelbine $25 \mathrm{mg} \mathrm{m}^{-2}$ 30-min intravenous infusion, and high-dose 5-FU $2600 \mathrm{mg} \mathrm{m}^{-2}$ plus LV $300 \mathrm{mg} \mathrm{m}^{-2}$ 24-h intravenous infusion (HDFL regimen) were given on days I and 8 every 3 weeks. Between June 1999 and April 2003, 40 patients with histologically confirmed recurrent or metastatic breast cancer were enrolled with a median age of 49 years (range: 36-68). A total of 25 patients had recurrent ABC, and I 5 patients had primary metastatic diseases. The overall response rate for the intent-to-treat group was 70.0\% (95\% Cl: 54-84\%) with eight complete responses and 20 partial responses. All 40 patients were evaluated for survival and toxicities. Among a total of 316 cycles of VNB-HDFL given (average: 7.9: range: 4- 14 cycles per patient), the main toxicity was Gr3/4 leucopenia and Gr3/4 neutropenia in 57 (I8.0\%) and 120 (38.0\%) cycles, respectively. Grl/2 infection and Grl/2 stomatitis were noted in five (I.6\%) and 59 (I8.7\%) cycles, respectively. None of the patients developed Gr3/4 stomatitis or Gr3/4 infection. Gr2/3 and Grl hand-foot syndrome was noted in two (5.0\%) and 23 (57.5\%) patients, respectively. Grl sensory neuropathy developed in three patients. The median time to progression was 8.0 months (range: $3-25.5$ months), and the median overall survival was 25.0 months with a followup of 5.5 to $45+$ months. This VNB-HDFL regimen is a highly active yet well-tolerated first-line treatment for $A B C$.

British Journal of Cancer (2005) 92, 1013 - 1018. doi:10.1038/sj.bjc.6602469 www.bjcancer.com

Published online 15 March 2005

(c) 2005 Cancer Research UK

Keywords: weekly vinorelbine; high-dose 5-FU and leucovorin; breast cancer

Breast cancer is the most common malignancy worldwide and is the fifth cancer killer in Taiwanese women. Despite adequate primary treatment with surgical resection and adjuvant chemohormonal therapy, about $25-30$ and $75-80 \%$ of patients without and with axillary node involvement at mastectomy, respectively, will have recurrent and/or metastatic breast cancer within 10 years of surgery (EBCTCG, 1992a, b, 1998; Horton, 1996). Combination chemotherapy will induce an objective response in approximately two-thirds of patients previously unexposed to chemotherapy, but complete eradication of disease at all sites will occur in less than 20\% (Ross et al, 1985; Clavel and Catimel, 1993).

*Correspondence: Dr AL Cheng, Department of Internal Medicine and Department of Oncology, National Taiwan University Hospital, No. 7, Chung-Shan South Road, Taipei 100, Taiwan;

E-mail: andrew@ha.mc.ntu.edu.tw

This study was presented in part to the 36th Annual Meeting of the American Society of Clinical Oncology (abstract 2340), Orlando, May 18-23, 2002.

Revised 22 November 2004; accepted 24 January 2005; published online I5 March 2005
Among the active chemotherapeutic agents in the treatment of breast cancer, alkylating agents, anthracyclines, and taxanes are the main components of most standard regimens. Anthracyclinebased combinations represent a highly active cytotoxic chemotherapeutic approach to the treatment of advanced breast cancer in the pre-taxane era (Ross et al, 1985: Clavel and Catimel, 1993). They are capable of inducing objective responses in $40-70 \%$ of patients (Ross et al, 1985; Clavel and Catimel, 1993). Further, taxane-based combinations also become major regimens for advanced breast cancer (Fornier et al, 2001; Buzdar et al, 2002; Davidson, 2002; Henderson et al, 2003). Among the active agents for advanced breast cancer, vinorelbine is also highly active both as a single agent and in combination regimens (Krikorian and Breillout, 1991).

Vinorelbine, a new semisynthetic vinca alkaloid, is well tolerated with significantly less neurotoxicity than other vinca alkaloids (vincristine and vinblastine) and a low incidence of subjective toxicities (Krikorian and Breillout, 1991). Vinorelbine, administered weekly as a single agent by an intravenous route, resulted in major objective response in about $45 \%$ of patients. Even in patients previously exposed to standard chemotherapy, $20-30 \%$ achieved a major objective response (Abeloff, 1995; Fumoleau et al, 1995; Hortobagyi, 1995; Smith, 1995). It is anticipated that vinorelbine 
will be increasingly utilised for treating metastatic breast cancer due to its favourable safety profile, good tolerability, and promising results in combination with other chemotherapy agents.

A phase I study suggested that the dose-limiting toxicity of vinorelbine was leucopenia and primarily neutropenia, and the maximum tolerated dose in single-agent therapy was $35.4 \mathrm{mg} \mathrm{m}^{-2}$ weekly (Mathe and Reizenstein, 1985). A series of phase II studies have shown that vinorelbine is a highly effective agent in the treatment of advanced breast cancer (Canobbio et al, 1989; Fumoleau et al, 1993; Garcia-Conde et al, 1994; Bruno et al, 1995). As first-line chemotherapy of advanced breast cancer, vinorelbine was studied in 4 phase II studies including 258 patients at a dose of $30 \mathrm{mg} \mathrm{m}^{-2}$ weekly (Canobbio et al, 1989; Fumoleau et al, 1993; Garcia-Conde et al, 1994; Bruno et al, 1995). The overall response rate was between $44 \%$ and $60 \%$. The median duration of response was between 17.9 and 36 weeks, and median survival ranged from 50.3 to 73 weeks.

Vinorelbine was also used in combination with other chemotherapy agents in first-line treatment for advanced breast cancer. In phase II studies, the combination of doxorubicin (50 $\mathrm{mg} \mathrm{m}^{-2}$ per cycle) (Spielmann et al, 1994) or epirubicin $\left(90 \mathrm{mg} \mathrm{m}^{-2}\right.$ per cycle) (Baldini et al, 1998) with $25 \mathrm{mg} \mathrm{m}^{-2}$ of vinorelbine on days 1 and 8 every 3 weeks resulted in a very impressive activity with an overall response rate of $74 \%(21 \%$ complete response plus $53 \%$ partial response) (Spielmann et al, $1994)$ and $70.2 \%(8.5 \%$ complete response plus $61.7 \%$ partial response) (Baldini et al, 1998), respectively. However, dose-limiting grade 4 neutropenia (70\%) (Baldini et al, 1998) and treatment-related grade 2-4 cardiotoxicity (10\%) (Spielmann et al, 1994) limited the clinical use of these combination regimens.

Searching for an effective yet well-tolerated regimen is mandatory. Among them, 5-fluorouracil (5-FU) is a potentially relevant candidate agent. 5-Fluorouracil could inhibit thymidylate synthase (TS) by 5-fluoro-2'-deoxyuridine monophosphate (FdUMP), a major metabolite of 5-FU, which binds to TS and prevents the formation of thymidine monophosphate (dTMP), which is an important precursor of thymidine triphosphate (dTTP), one of the four deoxynucleotides required for DNA synthesis (Grem et al, 1987; Bertino, 1997). Biochemical modulation of 5-FU by the addition of leucovorin (LV) results in the stabilisation of a ternary complex among FdUMP, LV, and TS. This enhances the inhibition of TS, the target enzyme of 5-FU (Grem et al, 1987; Bertino, 1997). Biochemical modulation of 5FU by LV has been shown to have a better response than 5-FU alone in colorectal cancer (ACCMAP, 1992). A similar modulation effect of LV on 5-FU has also been shown in breast cancer (Fine et al, 1994).

In advanced breast cancer, in a phase II study of vinorelbine administered at an intended dose of $30 \mathrm{mg} \mathrm{m}^{-2}$ on days 1 and 5 in combination with 5 -FU continuous infusion $\left(750 \mathrm{mg} \mathrm{m}^{-2}\right.$ daily for 5 days) every 3 weeks, an overall response rate of $61.6 \%$ was observed (Dieras et al, 1996a). The main toxicities (grades 3 and 4) were neutropenia ( $90 \%$ of patients), infection (12.7\%), and mucositis $(37 \%)$. The median response duration and overall survival were 12.3 and 23 months, respectively. Nole et al (1997) confirmed similar data in combination with vinorelbine, 5-FU, and $\mathrm{LV}$ with a response rate of $62 \%$. However, the 5-day loading schedule of 5 -FU $\left(50 \mathrm{mg} \mathrm{m}^{-2}\right.$ per day for 5 consecutive days as a continuous infusion) had significant treatment-related toxicity (such as $90 \%$ grade $3 / 4$ neutropenia and $37 \%$ grade $3 / 4$ mucositis, respectively), which may limit its clinical use (Dieras et al, 1996a). Searching for an effective yet well-tolerated combination is still mandatory.

Previously, we demonstrated that weekly 24-h infusion of high-dose 5-FU and LV (folinic acid), the HDFL regimen originally described by Ardalan et al (1991), appears to be particularly useful in gastric cancer (Hsu et al, 1997; Yeh and Cheng, 1998) and colon cancer (Yeh et al, 1997). We have provided evidence that prolonged exposure of gastric cancer cells to low concentration 5-FU for $24 \mathrm{~h}$ enhances the inhibition of TS, and thereby increases the cytotoxicity of 5-FU (Yeh et al, 2000b). Further, an HDFL regimen has repeatedly been demonstrated to cause minimal myelosuppression and therefore is an ideal component for combination chemotherapy with other cytotoxic agents against gastric cancer (Hsu et al, 1997; Yeh et al, 1997; Yeh and Cheng, 1998). We have also reported on the underlying mechanism of the low myelotoxicity of HDFL (Yeh et al, 2000a).

In one of our pilot studies, we used an HDFL regimen for the treatment of advanced breast cancer patients with heavily pretreated status or recurrence after high-dose chemotherapy with peripheral stem cells support. Even in this group of patients, an impressive response rate of $33 \%$ was noted.

In this study, we investigated if combination of weekly vinorelbine and weekly HDFL could be an effective yet welltolerated regimen of the first-line treatment for patients with advanced breast cancer.

\section{PATIENTS AND METHODS}

\section{Patients}

The chemotherapy was used as the first-line therapy, while previous postmastectomy adjuvant therapy (e.g., CMF regimens, anthracycline (doxorubicin, epirubicin)-based regimens, or lower dose 5-FU ( $\leqslant 750 \mathrm{mg} \mathrm{m}^{-2}$ per week)) given beyond 6 months before study enrolment was acceptable.

Eligibility criteria included (1) pathologically confirmed, recurrent or metastatic breast cancer, (2) at least one bidimensional measurable lesion on imaging studies, (3) ECOG performance status $\leqslant 2$, (4) age between 18 and 75 years, (5) adequate hepatic, renal, and bone marrow functions, and (6) fasting serum TG (1 day before the first cycle of chemotherapy) $>70 \mathrm{mg} \mathrm{dl}^{-1}$. The lower limit for serum TG was set to avoid HDFL-related hyperammonemic encephalopathy, which occurs in around 5\% of Taiwanese patients (Yeh and Cheng, 1997). Fasting serum TG level $\leqslant 70 \mathrm{mg} \mathrm{dl}^{-1}$ is the most important risk factor for HDFL-related hyperammonemic encephalopathy.

Exclusion criteria included (1) previous treatment with high-dose 5-FU ( $\geqslant 2000 \mathrm{mg} \mathrm{m}^{-2}$ per week), (2) prestudy fasting serum TG level $\leqslant 70 \mathrm{mg} \mathrm{dl}^{-1}$, (3) pregnant, breast-feeding, or woman of child-bearing potential without adequate contraception, (4) patients who refused placement of a central venous indwelling catheter (Port-A catheter) for outpatient chemotherapy, (4) concurrent or prior malignancy except curatively resected cervical carcinoma in situ or squamous cell carcinoma of skin, (5) central nervous system metastases, (6) active infection, and (7) concurrent treatments that could interfere with the study evaluation. This study was approved by the ethics committee of National Taiwan University Hospital and Changhua Christian Hospital. Signed informed consent was obtained from all patients.

\section{Study design}

This was a prospective phase II clinical trial.

\section{Chemotherapy protocol}

On days 1 and 8 of each cycle of chemotherapy, vinorelbine $25 \mathrm{mg} \mathrm{m}^{-2}$ was given as a 30 -min intravenous infusion, and followed by 5 -FU $2600 \mathrm{mg} \mathrm{m}^{-2}$ and LV (folinic acid) $300 \mathrm{mg} \mathrm{m}^{-2}$ given as a continuous 24 -h intravenous infusion. Treatment was repeated every 21 days. Treatment consisted of at least two cycles unless rapid disease progression occurred during treatment. 
Response assessment was performed every two cycles. Patients with complete response, partial response or stable disease (SD) continued the protocol treatment until intolerable toxicity. Patients with progressive disease were removed from the protocol treatment, and received other salvage treatment under the discretion of the responsible physicians. The response rate reported was the best tumour response obtained by the study treatment. Tumour response to the salvage therapy has not been included in the evaluation of the response rate.

\section{Dose modification}

For the first cycle of vinorelbine-HDFL, the following criteria were necessary: WBC count $\geqslant 4000 \mu \mathrm{l}^{-1}$ or ANC $\geqslant 2000 \mu \mathrm{l}^{-1}$, platelet count $\geqslant 100000 \mu \mathrm{l}^{-1}$, serum creatinine $\leqslant 1.5 \mathrm{mg} \mathrm{dl}^{-1}$, normal serum bilirubin level, and transaminases (AST or ALT) $\leqslant 3.5$-fold of the upper normal limits (UNLs) of reference values.

Patients were treated with the next cycle of vinorelbine-HDFL on day 22 or within a 3-week interval from the previous cycle of vinorelbine-HDFL. No dose reduction for vinorelbine was allowed. However, in cases of haematological, hepatic, or neurological toxicity, schedule modification was recommended as follows.

\section{Haematological toxicity}

Schedule modification for vinorelbine was based on blood count results obtained within 2 days of treatment, according to the following schedule: if leucopenia and/or thrombocytopenia $\leqslant$ grade 2 (WBC count $\geqslant 2000 \mu \mathrm{l}^{-1}$ and platelet count $\geqslant 50000 \mu \mathrm{l}^{-1}$ ), no schedule delay was indicated; and if leucopenia or thrombocytopenia $\geqslant$ grade 3 (WBC count $<2000 \mu \mathrm{l}^{-1}$ or platelet count $\left.<50000 \mu \mathrm{l}^{-1}\right)$, schedule delay and reassessment were indicated. If the study treatment could not be administered after a 3-week interval because of haematological toxicity, the patient was removed from protocol treatment.

\section{Neurological toxicity}

If peripheral neuropathy $\geqslant$ grade 2 , schedule delay and reassessment were indicated. If the study treatment could not be administered after a 3-week interval because of neurological toxicity, the patient was removed from protocol treatment.

Patients with pretreatment (before the first cycle of vinorelbineHDFL) fasting serum TG level $\leqslant 70 \mathrm{mg} \mathrm{dl}^{-1}$ were not neligible for this trial (Yeh and Cheng, 1997). If patients developed $\geqslant$ grade 2 toxicity of state of consciousness, HDFL was discontinued immediately until complete recovery was achieved under the best supportive care (Yeh and Cheng, 1997). Upon the altered state of consciousness, plasma ammonia level, lactic acid level, arterial blood gas, and complete biochemical screening (including the TG level) were immediately checked and the extent of HDFL-related neurotoxicity was documented. In the subsequent cycles, a $40 \%$ dose reduction for both 5 -FU and LV was indicated.

\section{Hepatic toxicity}

If total bilirubin or AST/ALT levels were abnormal in the absence of progressive disease, the following dose modifications were applied to vinorelbine: $(1) \leqslant$ grade 2 toxicity, that is, total bilirubin or AST/ALT was 1.26- to 5.0-fold of the UNLs of reference values, no dose modification was indicated, (2) grade 3 toxicity, that is, total bilirubin or AST/ALT was 5.1- to 10.0-fold of the UNLs, schedule delay and reassessment were indicated; and (3) grade 4 toxicity, that is, total bilirubin or AST/ALT was more than 10.0fold of the UNLs, vinorelbine was discontinued in the subsequent cycles.

\section{Other toxicities}

When $\geqslant$ grade 3 diarrhoea or stomatitis developed, HDFL chemotherapy was temporarily stopped for schedule delay and was reused after diarrhoea and stomatitis subsided to $\leqslant$ grade 1 .

\section{Evaluation of efficacy and toxicities}

Evaluations before chemotherapy included medical history taking, physical examination, complete blood count, blood chemistry, chest X-ray, and computed tomography (CT) scan of chest or abdomen as indicators of the location of lesions. After starting protocol treatment, complete blood count was examined weekly and blood chemistry every 2 weeks. The patients' condition and treatment-related toxicities were evaluated weekly. Tumour size was measured by imaging studies every two cycles, or when there were any clinical signs of possible tumour progression. Tumour response was evaluated according to the World Health Organization (WHO) criteria. Toxicities were graded using the NCIcommon toxicity criteria (version 2.0 ).

\section{Statistical methods}

This was a phase II, two-institutional, prospective study. The Simon two-stage design was used. The response rates of interest were $P_{0}=50 \%$ and $P_{1}=70 \%$. If there were more than 11 responders of the 23 patients in the first stage, the study then continued to enrol a total of 40 patients. If there were more than 24 responders of the 40 patients, this treatment would be acceptable with $\alpha$ of 0.10 and $\beta$ of 0.10 . Patients evaluable for response were those who had finished at least two cycles of treatment. All enrolled patients were subjected to toxicity evaluation.

Time to progression was defined as the duration from the date of starting protocol treatment to the date of documented disease progression or death by any cause. Overall survival was defined as the duration from the date of starting protocol treatment to the date of death. The Kaplan-Meier method was used in all survival analyses.

\section{RESULTS}

\section{Patients and treatment}

Between June 1999 and April 2003, 40 patients were enrolled in the study. The major clinicopathologic features of the patients are listed in Table 1. The median age was 49 years (range: $36-68$ ). A total of 25 patients had recurrent advanced breast cancer (15 had prior anthracycline-containing adjuvant chemotherapy, two had CMF adjuvant chemotherapy), and 15 patients had primary metastatic diseases. A total of $10(25 \%)$ patients had bone/soft tissue disease, and $10(25 \%)$ patients had three or more sites of metastatic diseases. Eight (20\%) patients had had postmastectomy adjuvant chemotherapy for more than 2 years; although they were not chemonaive patients, the study regimens for them were the first-line treatment after metastasis or recurrence.

A total of 316 cycles (median: 8; mean: 7.9; range: 4-14) of chemotherapy were given. In all, $107(33.9 \%)$ cycles needed a median schedule delay of 7 days (range: $3-14$ days) mainly due to grade $3 / 4$ neutropenia.

\section{Efficacy}

All 40 patients were evaluated for response. There were 16 responses in 23 patients in the first stage. Therefore, the study continued to enrol a total of 40 patients as scheduled by the twostage design. None of the patients had early progression or excluded from analysis for tumour response. There were eight 
Table I Clinicopathologic features of the patients

\begin{tabular}{lc}
\hline & Patient number \\
\hline Total patients & 40 \\
Age, median (range) & $49(36-68)$ \\
ECOG performance: & \\
0 & $10(25.0 \%)$ \\
1 & $24(60.0 \%)$ \\
2 & $6(15.0 \%)$ \\
Menopausal status & \\
Premenopause & $18(45.0 \%)$ \\
Postmenopause & $22(55.0 \%)$ \\
Oestrogen receptor status & \\
Positive & $21(52.5 \%)$ \\
Negative & $11(27.5 \%)$ \\
Unknown & $8(20.0 \%)$ \\
Disease status & \\
Recurrence/metastasis & $25(62.5 \%)$ \\
de novo metastasis & $15(37.5 \%)$ \\
Prior therapy & \\
Anthracycline-containing adjuvant chemotherapy & $15(37.5 \%)$ \\
CMF adjuvant chemotherapy & $2(5.0 \%)$ \\
Hormonal therapy & $21(52.5 \%)$ \\
Local radiotherapy & $11(27.5 \%)$ \\
Disease sites & \\
Lymph nodes & $33(82.5 \%)$ \\
Lung & $26(65.0 \%)$ \\
Breast & $19(47.5 \%)$ \\
Bone or/and spine & $16(40.0 \%)$ \\
Liver & $12(30.0 \%)$ \\
Pleural effusion & $8(20.0 \%)$ \\
Skin & $6(15.0 \%)$ \\
Others & $1(2.5 \%)$ \\
\hline
\end{tabular}

patients with complete remission (CR), 20 patients with partial remission (PR), and 12 patients with $\mathrm{SD}$. The overall response rate for the intent-to-treat group was 70.0\% (95\% confidence interval: $54-84 \%)$. The median time to tumour response was 1.5 months (range: 1.5-3.5 months). Four patients (10\%) dropped out after completion of two, four, six, and 12 cycles of study treatment in response conditions (one CR and three PR) due to treatment refusal. All of them were included in the survival analysis. None of the enrolled patients lost to follow-up.

Salvage therapy after disease progression included anthracycline-containing regimens, taxane-based regimens, trastuzumab, and aromatase inhibitors (anastrozole or letrozole) in nine, 19, six, and 11 patients, respectively. The median follow-up time of the whole group of 40 patients was 27 months. The median time to progression was 8.0 months (range: 3-25.5 months) (Figure 1). The median overall survival was 25 months (range: 5.5 to $45+$ months) (Figure 2).

\section{Toxicity}

All 40 patients were evaluated for toxicities (Table 2). The main toxicity was grade $3 / 4$ leucopenia and grade $3 / 4$ neutropenia in 57 $(18.0 \%)$ and $120(38.0 \%)$ cycles, respectively. Grade $1 / 2$ infection and grade $1 / 2$ stomatitis were noted in five (1.6\%) and $59(18.7 \%)$ cycles, respectively. None of the patients developed grade $3 / 4$ stomatitis or grade $3 / 4$ infection. Grade $3 / 4$ and grade $1 / 2$ diarrhoea was noted in $1(0.3 \%)$ and $26(8.2 \%)$ cycles, respectively. Grade $2 / 3$ and grade 1 hand-foot syndrome was noted in two $(5.0 \%)$ and $23(57.5 \%)$ patients, respectively. One patient needed a $25 \%$ dose reduction of $5-\mathrm{FU}$ due to grade 4 diarrhea, and one patient needed a $40 \%$ dose reduction of $5-\mathrm{FU} / \mathrm{LV}$ due to hyperammonemic encephalopathy during the first cycle. Grade 1 sensory neurotoxicity developed in three patients. Other nonhaematological toxicities were negligible. No patients discontinued

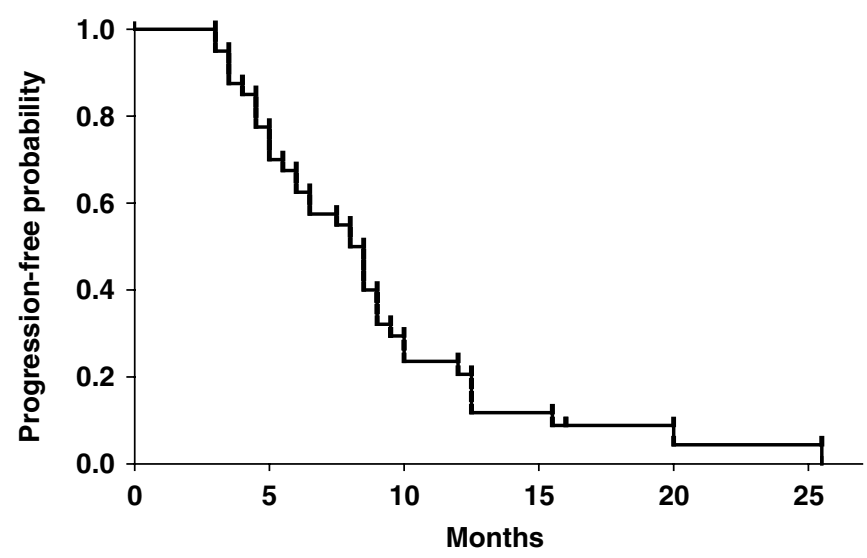

Figure I Kaplan-Meier curve for time-to-progression of the 40 patients.

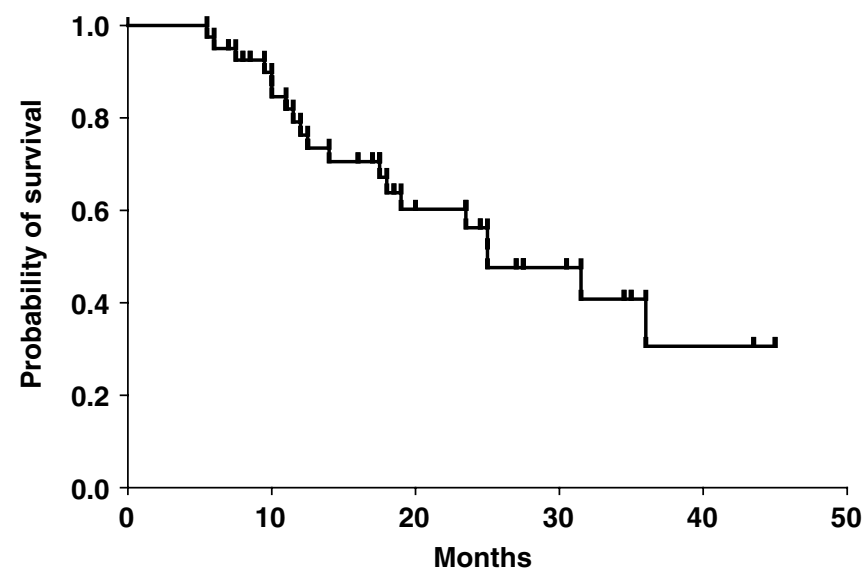

Figure 2 Kaplan-Meier curve for overall survival of the 40 patients.

Table 2 Toxicity of the vinorelbine-HDFL regimen

\begin{tabular}{|c|c|c|c|c|}
\hline \multirow[b]{2}{*}{ Toxicity } & \multicolumn{2}{|c|}{ Patients $(n=40)$} & \multicolumn{2}{|c|}{ Cycles $(n=316)$} \\
\hline & Grade I-2 & Grade 3-4 & Grade I-2 & Grade 3-4 \\
\hline \multicolumn{5}{|l|}{ Haematological } \\
\hline Neutropenia & $17.5 \%$ & $80.0 \%$ & $37.7 \%$ & $38.0 \%$ \\
\hline Leucopenia & $47.5 \%$ & $40.0 \%$ & $48.2 \%$ & $18.0 \%$ \\
\hline Thrombocytopenia & $10.0 \%$ & 0 & $2.5 \%$ & 0 \\
\hline Febrile neutropenia & - & $2.5 \%$ & - & $0.3 \%$ \\
\hline \multicolumn{5}{|l|}{ Gastrointestinal } \\
\hline Nausea & $80.0 \%$ & $5.0 \%$ & $49.4 \%$ & $1.6 \%$ \\
\hline Vomiting & $47.5 \%$ & $10.0 \%$ & $18.4 \%$ & $2.2 \%$ \\
\hline Diarrhoea & $35.0 \%$ & $2.5 \%$ & $8.2 \%$ & $0.3 \%$ \\
\hline Stomatitis & $55.0 \%$ & 0 & $18.7 \%$ & 0 \\
\hline Neuropathy & $7.5 \%$ & 0 & $3.8 \%$ & 0 \\
\hline Hepatic & $67.5 \%$ & $2.5 \%$ & $28.8 \%$ & $0.3 \%$ \\
\hline \multicolumn{5}{|l|}{ Others } \\
\hline Hand-foot syndrome & $57.5 \%^{\mathrm{a}}$ & $5.0 \%^{\mathrm{b}}$ & NA & NA \\
\hline Infection & $5.0 \%$ & 0 & $1.6 \%$ & 0 \\
\hline Alopecia & $75.0 \%$ & $2.5 \%$ & $54.7 \%$ & $0.6 \%$ \\
\hline
\end{tabular}

${ }^{a}$ Grade $1 .{ }^{b}$ Grade $2-3 . N A=$ nonapplicable by cycles

protocol treatment because of vinorelbine-related neuropathy. Due to cautious prestudy screening of fasting serum TG level and meticulous guidelines for dose modification of 5-FU and LV, only one of the patients developed HDFL-related hyperammonemic encephalopathy (Yeh and Cheng, 1997). 


\section{DISCUSSION}

The results of this phase II study indicated that this vinorelbineHDFL regimen using weekly vinorelbine and weekly 24 -h infusion of high-dose 5-FU/LV is effective for the treatment of advanced breast cancer. The overall response rate of $70.0 \%$ (95\% confidence interval: $54-84 \%)$ was within the range $(40-70 \%)$ of previously reported major protocols of anthracycline-based regimens (Ross et al, 1985; Clavel and Catimel, 1993; Jassem et al, 2001; Biganzoli et al, 2002; Sledge et al, 2003), taxane-based regimens (Jassem et al, 2001; Biganzoli et al, 2002; Sledge et al, 2003), and anthracyclineand-taxane-based regimens (Razis and Fountzilas, 2001; Sledge et al, 2003). Previously, studies from other series using vinorelbine and different administration schedules of 5-FU and LV have also demonstrated a good efficacy in breast cancer (Dieras et al, 1996a; Nole et al, 1997). However, the 5-day loading schedule of 5-FU created significant treatment-related toxicity, which may limit its clinical use (Dieras et al, 1996a). We report that this vinorelbineHDFL regimen using a unique schedule of 5-FU and LV is an effective yet well-tolerated combination for advanced breast cancer.

In this study, 5-FU and LV were given in two doses of weekly 24$\mathrm{h}$ infusion (HDFL regimen) every 3 weeks. The rationale for this scheduling of 5-FU/LV was based on our previous studies, which indicated that HDFL is in general a highly effective and very safe regimen for advanced gastric cancer (Hsu et al, 1997; Yeh and Cheng, 1998) and colorectal cancer (Yeh et al, 1997). In our pilot studies, we used an HDFL regimen for the treatment of advanced breast cancer patients with heavily pretreated status or recurrence after high-dose chemotherapy. Even in this group of patients, an impressive response rate of $33 \%$ was noted.

The patients' compliance with this HDFL regimen was generally good. Further, results of our in vitro studies have implied that strict avoidance of bolus injection of 5-FU is the key to avoiding myelosuppression (Yeh et al, 2000a). Although the best protocol of 5-FU remains to be explored (Bertino, 1997; Grem, 2001), both clinical and laboratory data indicate that HDFL is an ideal component for combination chemotherapy with other cytotoxic agents. For instance, paclitaxel followed by weekly high-dose 5-FU and LV infusion has been shown to have a 55\% response rate in anthracycline-resistant metastatic breast cancer patients (Klaassen et al, 1995, 1996). In this study, we showed that weekly vinorelbine plus weekly HDFL is an effective regimen with welltolerated toxicities for the first-line treatment of advanced breast cancer.

Although survival is not a major end point for evaluation of efficacy in phase II trials, the median overall survival of 25 months in this study was favourably within the range of other previously reported major protocols of anthracycline-based regimens (Ross et al, 1985; Clavel and Catimel, 1993; Jassem et al, 2001; Biganzoli et al, 2002; Sledge et al, 2003), taxane-based regimens (Jassem et al, 2001; Biganzoli et al, 2002; Sledge et al, 2003), and vinorelbinebased regimens (Dieras et al, 1996a; Nole et al, 1997; Norris et al, 2000).

The toxicity of the current vinorelbine-HDFL protocol is generally well tolerated. Although vinorelbine-related neurotoxicity did occur in this study, it was usually mild (grade 1/2) under this weekly schedule. No patients discontinued protocol treatment due to vinorelbine-related neuropathy. In contrast, the paclitaxelrelated neuropathy was found to be one of the dose-limiting toxicities in paclitaxel-containing regimens for breast cancer (Postma et al, 1995; Forsyth et al, 1997; Gelmon et al, 1999). Other major toxicities, including diarrohea and stomatitis, were of lesser severity than for other major regimens using vinorelbine and a 5-day schedule of 5-FU and LV (Dieras et al, 1996a; Nole et al, 1997). Neutropenia and leucopenia were not less severe than other reported regimens using vinorelbine and 5-FU; however, the doses, schedules, and G-CSF support of these regimens were essentially different from the current regimen (Kornek et al, 1998; Lombardi et al, 2000). Regimen used by Kornek et al (1998) required G-CSF support at $5 \mu \mathrm{g} \mathrm{kg}^{-1}$ day $^{-1}$ subcutaneously on days $6-10$ during each cycle, and it was complicated with septicaemia in two patients. Regimen used by Lombardi et al (2000) applied lower dose intensity of vinorelbine $\left(20 \mathrm{mg} \mathrm{m}^{-2}\right.$ on days 1 and 8 , every 4 weeks) than current regimen $\left(25 \mathrm{mg} \mathrm{m}^{-2}\right.$ on days 1 and 8 , every 3 weeks). In addition, the regimen by Lombardi et al (2000) used protracted continuous infusion (PCI) of low-dose 5-FU $\left(250 \mathrm{mg} \mathrm{m}^{-2} \mathrm{day}^{-1}\right)$ (Lombardi et al, 2000), rather than a convenient weekly HDFL. Although grade $3 / 4$ neutropenia and grade 3/4 leucopenia were still common, the rarity of both grade $3 /$ 4 stomatitis and grade $3 / 4$ diarrohea may contribute to the absence of grade 3/4 infections in this study. Prophylactic G-CSF support was not used in this study. A total of 117 (33.9\%) cycles needed a median delay of 7 days (range: 3-14 days). Prophylactic antibiotics with oral quinolones were used in $7.5 \%$ of patients. Overall, the toxicity profiles of the current vinorelbine-HDFL protocol were generally well tolerated.

We conclude that combination of weekly vinorelbine and weekly 24-h infusion of high-dose 5-FU and LV is a highly effective regimen with well-tolerated toxicities for the first-line treatment of advanced breast cancer.

\section{ACKNOWLEDGEMENTS}

This study was supported by grants from the National Health Research Institutes (NHRI-CN-CA-9201S), Taiwan.

\section{REFERENCES}

Abeloff MD (1995) Vinorelbine (Navelbine) in the treatment of breast cancer: a summary. Semin Oncol 22(2 Suppl 5): 1-4

ACCMAP (1992) Modulation of fluorouracil by leucovorin in patients with advanced colorectal cancer: evidence in terms of response rate. Advanced Colorectal Cancer Meta-Analysis Project. J Clin Oncol 10: 896-903

Ardalan B, Chua L, Tian EM, Reddy R, Sridhar K, Benedetto P, Richman S, Legaspi A, Waldman S, Morrell L (1991) A phase II study of weekly 24hour infusion with high-dose fluorouracil with leucovorin in colorectal carcinoma. J Clin Oncol 9: 625-630

Baldini E, Tibaldi C, Chiavacci F, Di Lieto M, Fioretto L, Giallom-bardo A, Taviani R, Ghezzi P, Bolognini A, Conte P (1998) Epirubicin/vinorelbine as first line therapy in metastatic breast cancer. Breast Cancer Res Treat 49: $129-134$

Bertino JR (1997) Biomodulation of 5-fluorouracil with antifolates. Semin Oncol 24(5 Suppl 18): $52-56$
Biganzoli L, Cufer T, Bruning P, Coleman R, Duchateau L, Calvert AH, Gamucci T, Twelves C, Fargeot P, Epelbaum R, Lohrisch C, Piccart MJ (2002) Doxorubicin and paclitaxel versus doxorubicin and cyclophosphamide as first-line chemotherapy in metastatic breast cancer: The European Organization for Research and Treatment of Cancer 10961 Multicenter Phase III Trial. J Clin Oncol 20: 3114-3121

Bruno S, Puerto VL, Mickiewicz E, Hegg R, Texeira LC, Gaitan L, Martinez L, Fernandez O, Otero J, Kesselring G (1995) Phase II trial of weekly i.v. vinorelbine as a single agent in first-line advanced breast cancer chemotherapy. The Latin-American experience. Am J Clin Oncol 18: 392-396 Buzdar AU, Singletary SE, Valero V, Booser DJ, Ibrahim NK, Rahman Z, Theriault RL, Walters R, Rivera E, Smith TL, Holmes FA, Hoy E, Frye DK, Manuel N, Kau SW, McNeese MD, Strom E, Thomas E, Hunt K, Ames F, Berry D, Hortobagyi GN (2002) Evaluation of paclitaxel in adjuvant chemotherapy for patients with operable breast cancer: preliminary data of a prospective randomized trial. Clin Cancer Res 8: 1073-1079 
Canobbio L, Boccardo F, Pastorino G, Brema F, Martini C, Resasco M, Santi L (1989) Phase-II study of Navelbine in advanced breast cancer. Semin Oncol 16(2 Suppl 4): 33-36

Clavel M, Catimel G (1993) Breast cancer: chemotherapy in the treatment of advanced disease. Eur J Cancer 29A: 598-604

Davidson NE (2002) Ongoing US cooperative group trials using taxanes in the adjuvant setting. Clin Breast Cancer 3(Suppl 2): S53-S58

Dieras V, Extra JM, Bellissant E, Espie M, Morvan F, Pierga JY, Mignot L, Tresca P, Marty M (1996a) Efficacy and tolerance of vinorelbine and fluorouracil combination as first-line chemotherapy of advanced breast cancer: results of a phase II study using a sequential group method. J Clin Oncol 14: $3097-3104$

EBCTCG (1992a) Systemic treatment of early breast cancer by hormonal, cytotoxic, or immune therapy. 133 randomised trials involving 31,000 recurrences and 24,000 deaths among 75,000 women. Early Breast Cancer Trialists' Collaborative Group. Lancet 339: $71-85$

EBCTCG (1992b) Systemic treatment of early breast cancer by hormonal, cytotoxic, or immune therapy. 133 randomised trials involving 31,000 recurrences and 24,000 deaths among 75,000 women. Early Breast Cancer Trialists' Collaborative Group. Lancet 339: 1 - 15

EBCTCG (1998) Polychemotherapy for early breast cancer: an overview of the randomised trials. Early Breast Cancer Trialists' Collaborative Group. Lancet 352: $930-942$

Fine S, Erlichman C, Kaizer L, Warr D, Gadalla T (1994) Phase II trial of 5 -fluorouracil and folinic acid in the treatment of advanced breast cancer. Breast Cancer Res Treat 30: 205-209

Fornier MN, Seidman AD, Theodoulou M, Moynahan ME, Currie V, Moasser M, Sklarin N, Gilewski T, D'Andrea G, Salvaggio R, Panageas KS, Norton L, Hudis C (2001) Doxorubicin followed by sequential paclitaxel and cyclophosphamide versus concurrent paclitaxel and cyclophosphamide: 5-year results of a phase II randomized trial of adjuvant dose-dense chemotherapy for women with node-positive breast carcinoma. Clin Cancer Res 7: 3934-3941

Forsyth PA, Balmaceda C, Peterson K, Seidman AD, Brasher P, DeAngelis LM (1997) Prospective study of paclitaxel-induced peripheral neuropathy with quantitative sensory testing. J Neurooncol 35: 47-53

Fumoleau P, Delgado FM, Delozier T, Monnier A, Gil Delgado MA, Kerbrat P, Garcia-Giralt E, Keiling R, Namer M, Closon MT (1993) Phase II trial of weekly intravenous vinorelbine in first-line advanced breast cancer chemotherapy. J Clin Oncol 11: 1245-1252

Fumoleau P, Delozier T, Extra JM, Canobbio L, Delgado FM, Hurteloup P (1995) Vinorelbine (Navelbine) in the treatment of breast cancer: the European experience. Semin Oncol 22(2 Suppl 5): 22-28

Garcia-Conde J, Lluch A, Martin M, Casado A, Gervasio H, De Oliveira C, De Pablo JL, Gorostiaga J, Giron GC, Cervantes A (1994) Phase II trial of weekly IV vinorelbine in first-line advanced breast cancer chemotherapy. Ann Oncol 5: $854-857$

Gelmon K, Eisenhauer E, Bryce C, Tolcher A, Mayer L, Tomlinson E, Zee B, Blackstein M, Tomiak E, Yau J (1999) Randomized phase II study of high-dose paclitaxel with or without amifostine in patients with metastatic breast cancer. J Clin Oncol 17: 3038-3047

Grem JL (2001) Biochemical modulation of 5-FU in systemic treatment of advanced colorectal cancer. Oncology 15(1 Suppl 2): $13-19$

Grem JL, Hoth DF, Hamilton JM, King SA, Leyland-Jones B (1987) Overview of current status and future direction of clinical trials with 5fluorouracil in combination with folinic acid. Cancer Treat Rep 71: $1249-1264$

Henderson IC, Berry DA, Demetri GD, Cirrincione CT, Goldstein LJ, Martino S, Ingle JN, Cooper MR, Hayes DF, Tkaczuk KH, Fleming G, Holland JF, Duggan DB, Carpenter JT, Frei E, Schilsky RL, Wood WC, Muss HB, Norton L (2003) Improved outcomes from adding sequential paclitaxel but not from escalating doxorubicin dose in an adjuvant chemotherapy regimen for patients with node-positive primary breast cancer. J Clin Oncol 21: $976-983$

Hortobagyi GN (1995) Future directions for vinorelbine (Navelbine). Semin Oncol 22(2 Suppl 5): $80-86$

Horton J (1996) 1995 Oxford breast cancer overview - preliminary outcomes. Cancer Control 3: 78-79

Hsu CH, Yeh KH, Chen LT, Liu JM, Jan CM, Lin JT, Chen YC, Cheng AL (1997) Weekly 24-hour infusion of high-dose 5-fluorouracil and leucovorin in the treatment of advanced gastric cancers. An effective and low-toxic regimen for patients with poor general condition. Oncology 54: $275-280$

Jassem J, Pienkowski T, Pluzanska A, Jelic S, Gorbunova V, MrsicKrmpotic Z, Berzins J, Nagykalnai T, Wigler N, Renard J, Munier S, Weil
C (2001) Doxorubicin and paclitaxel versus fluorouracil, doxorubicin, and cyclophosphamide as first-line therapy for women with metastatic breast cancer: final results of a randomized phase III multicenter trial. J Clin Oncol 19: $1707-1715$

Klaassen U, Wilke H, Pari CP, Strumberg D, Harstrick A, Eberhardt W, Becher R, Diergarten K, Seeber S (1995) Phase I/II study with paclitaxel in combination with weekly high-dose 5-fluorouracil/folinic acid in the treatment of metastatic breast cancer: an interim analysis. Semin Oncol 22(6 Suppl 14): 7-11

Klaassen U, Wilke H, Seeber S (1996) Paclitaxel combined with weekly high-dose 5-fluorouracil/folinic acid and cisplatin in the treatment of advanced breast cancer. Semin Oncol 23(5 Suppl 11): 32-37

Kornek GV, Haider K, Kwasny W, Lang F, Krauss G, Hejna M, Raderer M, Weinlander G, Depisch D, Scheithauer W (1998) Effective treatment of advanced breast cancer with vinorelbine, 5-fluorouracil and l-leucovorin plus human granulocyte colony-stimulating factor. $\mathrm{Br} \mathrm{J}$ Cancer 78: $673-678$

Krikorian A, Breillout F (1991) Vinorelbine (Navelbine). A new semisynthetic vinca alkaloid. Onkologie 14: 7-12

Lombardi D, Magri MD, Crivellari D, Spazzapan S, Paolello C, De Cicco M, Di Lauro V, Scuderi C, Veronesi A (2000) Combination chemotherapy with navelbine and continuous infusion of 5-fluorouracil in metastatic, chemotherapy refractory breast cancer. Ann Oncol 11: $1041-1043$

Mathe G, Reizenstein P (1985) Phase I pharmacologic study of a new Vinca alkaloid: navelbine. Cancer Lett 27: 285-293

Nole F, de Braud F, Aapro M, Minchella I, De Pas M, Zampino MG, Monti S, Andreoni G, Goldhirsch A (1997) Phase I-II study of vinorelbine in combination with 5-fluorouracil and folinic acid as first-line chemotherapy in metastatic breast cancer: a regimen with a low subjective toxic burden. Ann Oncol 8: $865-870$

Norris B, Pritchard KI, James K, Myles J, Bennett K, Marlin S, Skillings J, Findlay B, Vandenberg T, Goss P, Latreille J, Rudinskas L, Lofters W, Trudeau M, Osoba D, Rodgers A (2000) Phase III comparative study of vinorelbine combined with doxorubicin versus doxorubicin alone in disseminated metastatic/recurrent breast cancer: National Cancer Institute of Canada Clinical Trials Group Study MA8. J Clin Oncol 18: $2385-2894$

Postma TJ, Vermorken JB, Liefting AJ, Pinedo HM, Heimans JJ (1995) Paclitaxel-induced neuropathy. Ann Oncol 6: 489-494

Razis ED, Fountzilas G (2001) Paclitaxel: epirubicin in metastatic breast cancer - a review. Ann Oncol 12: $593-598$

Ross MB, Buzdar AU, Smith TL, Eckles N, Hortobagyi GN, Blumenschein GR, Freireich EJ, Gehan EA (1985) Improved survival of patients with metastatic breast cancer receiving combination chemotherapy. Cancer 55: $341-346$

Sledge GW, Neuberg D, Bernardo P, Ingle JN, Martino S, Rowinsky EK, Wood WC (2003) Phase III trial of doxorubicin, paclitaxel, and the combination of doxorubicin and paclitaxel as front-line chemotherapy for metastatic breast cancer: an intergroup trial (E1193). J Clin Oncol 21: $588-592$

Smith GA (1995) Current status of vinorelbine for breast cancer. Oncology (Huntingt) 9: $767-773$

Spielmann M, Dorval T, Turpin F, Antoine E, Jouve M, Maylevin F, Lacombe D, Rouesse J, Pouillart P, Tursz T (1994) Phase II trial of vinorelbine/doxorubicin as first-line therapy of advanced breast cancer. J Clin Oncol 12: $1764-1770$

Yeh KH, Cheng AL (1997) High-dose 5-fluorouracil infusional therapy is associated with hyperammonaemia, lactic acidosis and encephalopathy. Br J Cancer 75: $464-465$

Yeh KH, Cheng AL (1998) Gastric cancer associated with acute disseminated intravascular coagulation: successful initial treatment with weekly 24 -hour infusion of high-dose 5 -fluorouracil and leucovorin. $\mathrm{Br} \mathrm{J}$ Haematol 100: 769-772

Yeh KH, Cheng AL, Lin MT, Hong RL, Hsu CH, Lin JF, Chang KJ, Lee PH, Chen YC (1997) A phase II study of weekly 24-hour infusion of high-dose 5-fluorouracil and leucovorin (HDFL) in the treatment of recurrent or metastatic colorectal cancers. Anticancer Res 17: $3867-3871$

Yeh KH, Yeh SH, Chang YS, Cheng AL (2000a) Minimal toxicity to myeloid progenitor cells of weekly 24-hr infusion of high-dose 5-fluorouracil: direct evidence from colony forming unit-granulocyte and monocyte (CFU-GM) clonogenic assay. Pharmacol Toxicol 86: 122-124

Yeh KH, Yeh SH, Hsu CH, Wang TM, Ma IF, Cheng AL (2000b) Prolonged and enhanced suppression of thymidylate synthase by weekly $24-\mathrm{h}$ infusion of high-dose 5-fluorouracil. Br J Cancer 83: 1510-1515 